\title{
Verification and Analysis of Single-Molecule SERS events via Polarization-Selective Raman Measurement
}

Qi HAO ${ }^{1,2,3}$, Zhaohui PENG ${ }^{1}$, Jiawei $W A N G^{3,4}$, Xingce FAN ${ }^{1}$, Guoqun $L I^{1}$, Xing

ZHAO', Libo MA ${ }^{3}$, Teng QIU ${ }^{1, *}$ and Oliver G. Schmidt ${ }^{3,5}$.

${ }^{1}$ School of Physics, Southeast University, Nanjing 211189, P. R. China

${ }^{2}$ Quantum Information Research Center, Southeast University, Nanjing 211189, P. R.

China;

${ }^{3}$ Institute for Integrative Nanosciences, Leibniz IFW Dresden, Helmholtzstraße 20, 01069, Germany

${ }^{4}$ Department of Electronic and Information Engineering, Harbin Institute of Technology (Shenzhen), Shenzhen 518055, P. R. China;

${ }^{5}$ Material Systems for Nanoelectronics, Technische Universität Chemnitz, 09111, Germany 


\section{Table of contents}

Supplementary Information I: Fabrication and plasmonic dimer arrays by using anodic

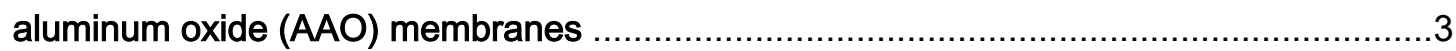

a. Fabrication of anodic aluminum oxide (AAO) membranes on aluminum foil. .......3

b. Fabrication of freestanding porous AAO membranes. ...................................

c. Fabrication of plasmonic dimers by using AAO templates in angle-resolved shadow deposition. .5

Supplementary Information II: Single-molecule SERS (SMSERS) detection. .5

a. Enhancement factor requirement for single-molecule SERS (SMSERS) detection 5

b. Polarization-selective Raman measurement (PRM) .6

c. FWHM analysis of the SMSERS events 6

References: .7 
Supplementary Information I: Fabrication and plasmonic dimer arrays by using anodic aluminum oxide (AAO) membranes

a. Fabrication of anodic aluminum oxide (AAO) membranes on aluminum foil.

The AAO membrane was formed on an aluminum foil (99.999\%, $0.2 \mathrm{~mm}$ in thickness) by using the two-step anodization method, see Figure S1. The aluminum foil was degreased with

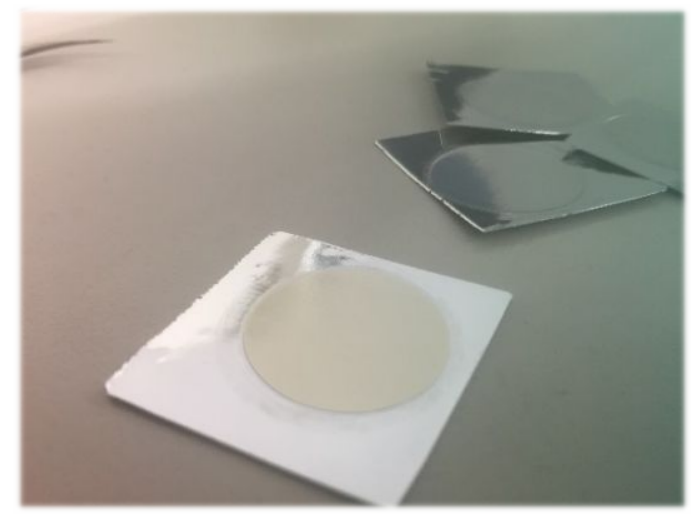

Figure S1. A photography figure of AAO membrane on aluminum film.

acetone, annealed at $400{ }^{\circ} \mathrm{C}$ for 30 min under vacuum to remove mechanical stress, and electropolished in a 1:4 solution of perchloric acid and ethanol at $15 \mathrm{~V}$ for $5 \mathrm{~min}$. The first anodization was performed by applying a DC voltage of $40 \mathrm{~V}$ in $0.3 \mathrm{M}$ oxalic acid at $0{ }^{\circ} \mathrm{C}$ for $12 \mathrm{~h}$. The alumina formed in the first anodization process was dissolved by chemical wet etching in a solution of phosphoric acid (6 wt \%) and chromic acid (1.8 wt \%) with a volume ratio of $1: 1$ at $75^{\circ} \mathrm{C}$ for $2 \mathrm{~h}$. The aluminum foil was subjected to a DC voltage of $40 \mathrm{~V}$ in the same solution as the first anodization step but for short anodization time of 5 min to produce the ultra-thin AAO membrane with a pore depth of $300 \mathrm{~nm}$, as shown in Figure S2.
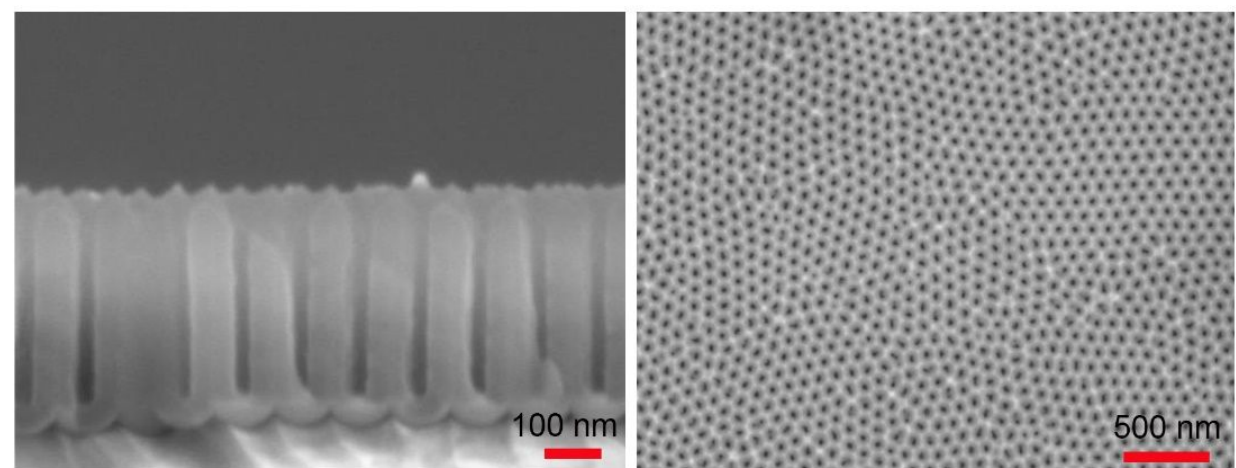

Figure S2. SEM images of the fabricated AAO membranes on aluminum foil. 

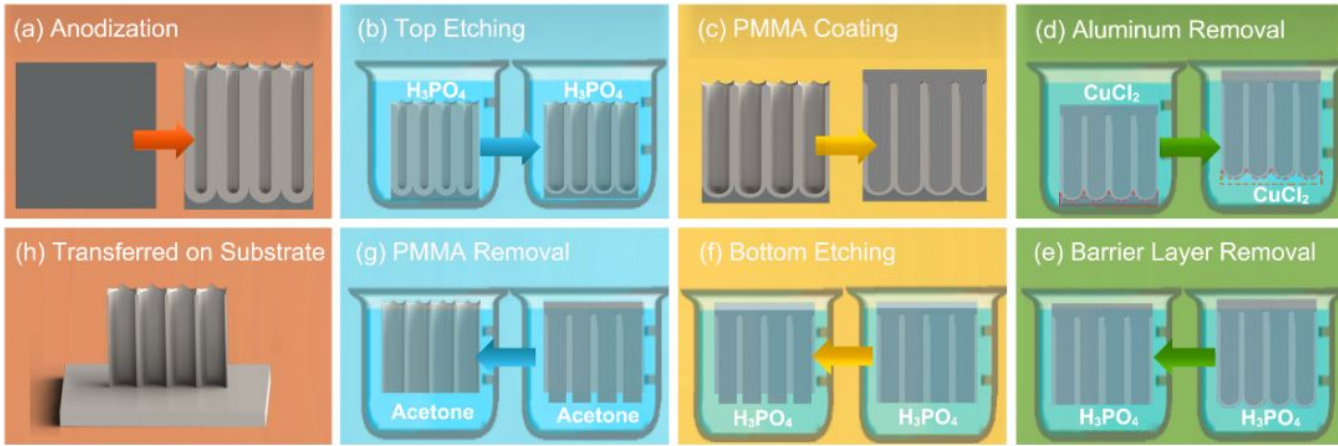

Figure S3. Schematic figures illustrating the fabrication of freestanding porous AAO membranes.

\section{b. Fabrication of freestanding porous AAO membranes.}

The schematic of this process was illustrated in Figure S3. After the anodization step, the AAO membranes were immersed in $5 \mathrm{wt} \%$ phosphoric acid at $30^{\circ} \mathrm{C}$ for $30 \mathrm{~min}$ for top etching to broaden the AAO pores from $35 \mathrm{~nm}$ to $80 \mathrm{~nm}$. Afterwards, The AAO was spin-coated with a PMMA layer (950PMMA A4 from MicroChem) at $600 \mathrm{rpm}$ for $8 \mathrm{~s}$ and $3000 \mathrm{rpm}$ for $60 \mathrm{~s}$ (Laurell spin coater WS-400BZ-6NPP-Lit). The PMMA/AAO membrane was annealed at $120{ }^{\circ} \mathrm{C}$ for 30 min to ensure that PMMA fill the pores. The remaining aluminum foil was etched in $100 \mathrm{~g} / \mathrm{L} \mathrm{CuCl}_{2}$ solution. Afterwards, AAO barrier layer was removed in a $5 \mathrm{wt} \%$ phosphoric acid solution for 25 min. The thickness of AAO can be precisely controlled in this process by controlling the etching time, which is essential for the angle-resolved deposition. Finally, the PMMA layer was dissolved in acetone and then the remaining AAO membrane was rinsed with distilled water and transferred onto a silicon wafer, see Figure $\mathrm{S} 4$. The wafers were cleaned with 5:1:1 $\mathrm{H}_{2} \mathrm{O} / \mathrm{NH}_{4} \mathrm{OH} / 30 \%$ $\mathrm{H}_{2} \mathrm{O}_{2}$ solution. It should be noted that AAO thickness decrease with the etching at a rate of about $1.5 \mathrm{~nm} / \mathrm{min}$. The final thickness of AAO membrane is $270 \mathrm{~nm} \pm 10 \mathrm{~nm}$ after etching.

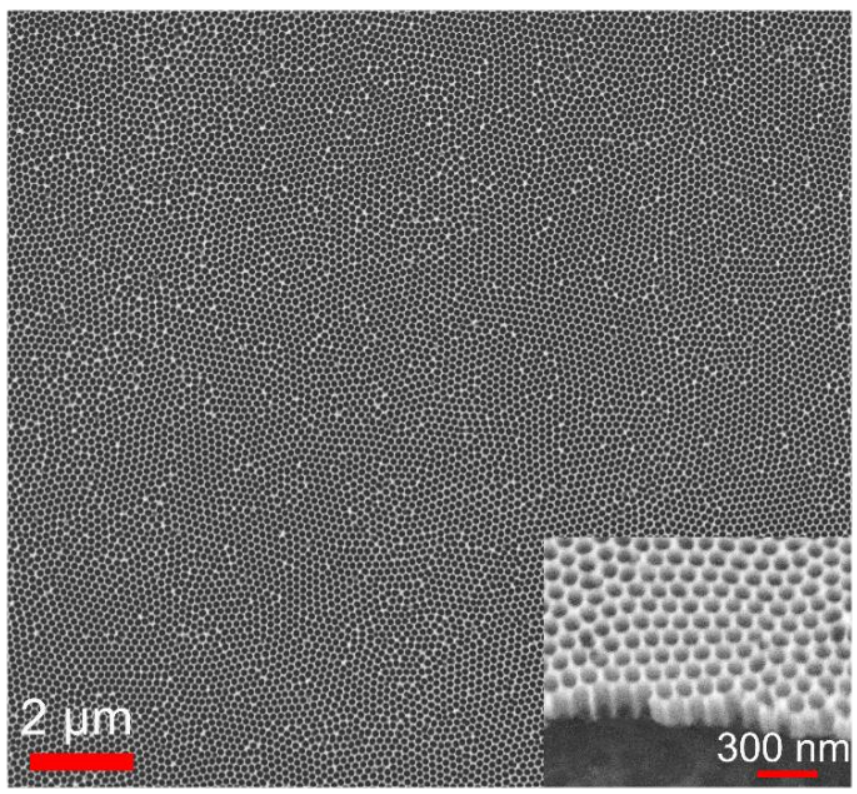

Figure S4. Top and side view SEM images of AAO transferred on silicon substrate. 
c. Fabrication of plasmonic dimers by using AAO templates in angle-resolved shadow deposition.

The porous AAO membranes with a pore diameter of $80 \mathrm{~nm}$ and a film thickness of $270 \mathrm{~nm}$ were employed to fabricate dimer arrays. The samples were placed on a tilted stage to perform angle-resolved shadow deposition, as shown in Figure S5. The E-beam evaporation was conducted for two times with opposite deposition angles of $\tan \alpha=1 / 5$ to fabricate plasmonic homodimers with an estimated dimer gap of $8 \mathrm{~nm}$. The E-beam deposition rate is $0.25 \mathrm{~nm} \mathrm{~s}^{-1}$ and the deposition thickness is $30 \mathrm{~nm}$. After deposition, the AAO masks were removed with a tape and the plasmonic dimers were obtained. This method can be extended to fabricated heterodimers with different materials ranging from metal, semiconductor to oxides and nitrides.
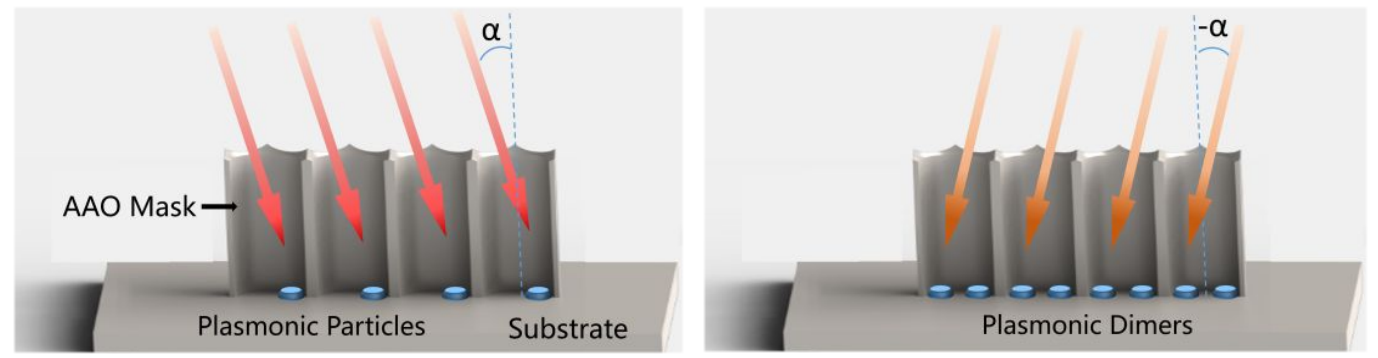

Figure S5. Schematic figures illustrating the fabrication of plasmonic dimers in shadow deposition.

\section{Supplementary Information II: Single-molecule SERS (SMSERS) detection}

\section{a. Enhancement factor requirement for single-molecule SERS (SMSERS) detection}

The calculated EM distributions around silver plasmonic dimers are presented in Figure S6. The distribution of EM fields is uniform across the surface of plasmonic materials. Therefore, the position of molecule is an important consideration for SMSERS. Generally, $\sim 80 \%$ of the SERS signal is generated by less than $0.1 \%$ of the molecules around the substrate. For SMSERS, a molecule is only detectable when it is located in a hot spot, and its signal intensity spans as high as 3 orders of magnitude due to the nonhomogeneous distribution of EM fields. The observation of a single-molecule by SERS requires an enhancement factor of $10^{14}-10^{16}$ considering the Raman cross section of molecules. Generally speaking, EM enhancement contributes the most, while chemically charge transfer offers an enhancement factor of $10^{2}$ $10^{3}$ and resonance enhancement may provide additional enhancement up to $10^{4}-10^{6}$ considering the matching between laser energy and molecular adsorption bands. In this article, the EM enhancement factors around the gap position excited by parallel polarized laser is about 2 orders of magnitude higher than the EFs under perpendicular excitation. 

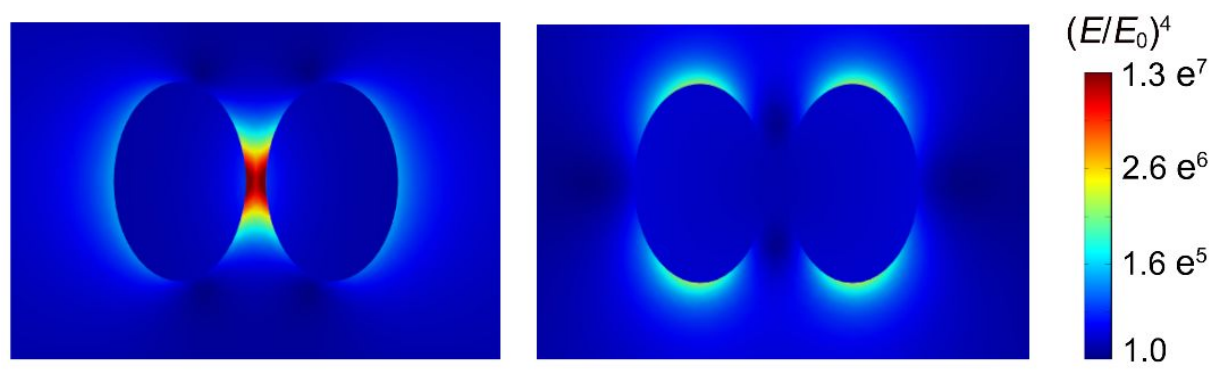

Figure S6. EM enhancement factor distribution around plasmonic dimers under parallel and perpendicular excitation calculated by finite element modeling (FEM) method. The long axis of a particle is $80 \mathrm{~nm}$ and the gap between dimers is $8 \mathrm{~nm}$. The excitation wavelength is $458 \mathrm{~nm}$.

\section{b. Polarization-selective Raman measurement (PRM)}

In our experiment, the molecular concentration of $\mathrm{R} 6 \mathrm{G}$ and $\mathrm{CV}$ is $10^{-11} \mathrm{M}$, the volume of the solution is $300 \mu \mathrm{L}$, the sample size is $1 \mathrm{~cm}^{2}$, the dimer density is $10^{10} / \mathrm{cm}^{2}$, and the laser spot is $\sim 800 \mathrm{~nm}$. According to the Langmuir's adsorption isotherm[1], only $40 \%$ to $50 \%$ molecule adsorbs to silver dimers under our experimental condition, corresponding to an average of $\sim 0.1$ molecules per dimer, namely $\sim 5$ molecules with in the spectroscopic volume. Considering that only $0.2 \%-1 \%$ of the surface area of plasmonic material is capable to provide single-molecule sensitivity, therefore the expected possibility to get SMSERS event in single measurement is $1 \%-5 \%$. The data implies that the probability to get few-molecule events in an individual SERS measurement is small. The value is higher than the experimental data $0.9 \%$. It may arise from the disturbance from signal blinking and overestimation of the number of molecules within the spectroscopic volume.

\section{c. FWHM analysis of the SMSERS events}
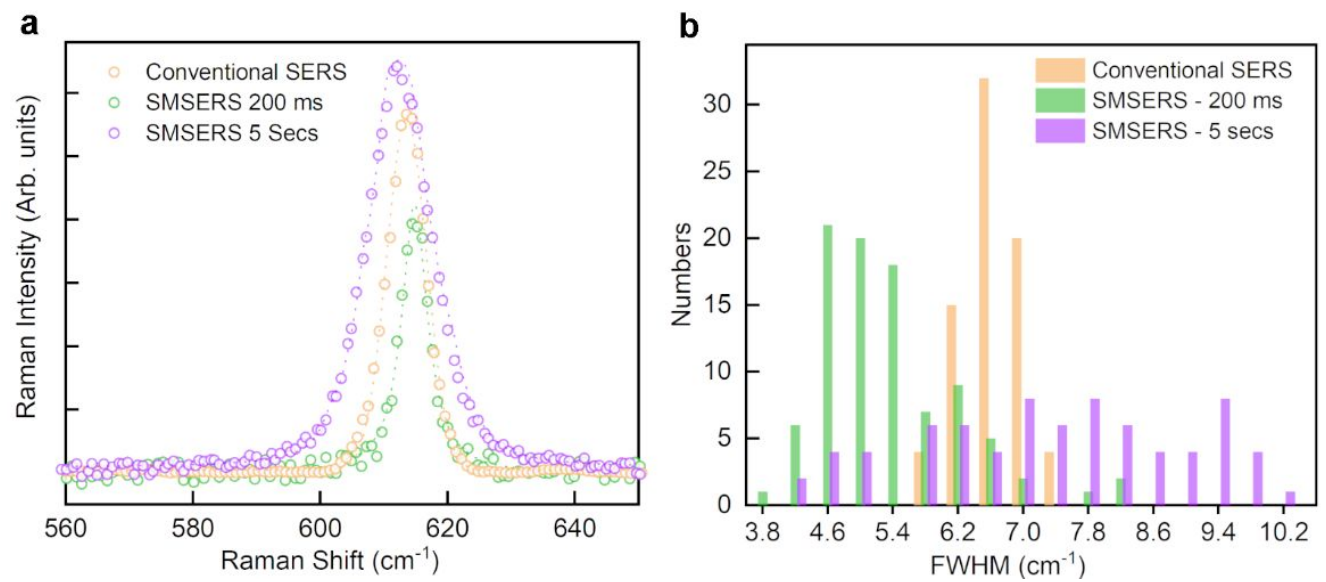

Figure S7. a) Typical ensemble SERS spectrum and SMSERS spectra with a collection time of $200 \mathrm{~ms}$ and 5 secs. b) FHHMs distribution of conventional SERS events and SMSERS events with a different collection time. 
Figure S7a presents typical ensemble SERS spectrum and SMSERS spectra with a collection time of $200 \mathrm{~ms}$ and 5 secs. The FWHMs of SMD events exhibit a strong dependence on the collection time. The narrowed FWHM is observed with a short timescale while a significant broadening arising from molecular diffusions is observed when the detection time is extended to 5 secs. This phenomenon differentiates SMSERS with ensemble SERS spectra, which is helpful for the confirmation of SMSERS events. The FWHMs distribution of conventional SERS events and SMSERS events are plotted in Figure S7b. It should be noted the data collects from random points to interpret the natural distribution of FWHMs. The FWHM of an individual SMSERS event is changing in multiple measurements and this is not considered in Figure S7b.

Figure S8 illustrates the FWHM distribution of SERS events with regard to the intensity ratio $f$ $=I_{0} / I_{\pi / 2}$, where $I_{0}$ and $I_{\pi / 2}$ represents the peak intensity of R6G $1650 \mathrm{~cm}^{-1}$ mode at $\theta=0$ and $\pi / 2$, respectively, or the noise power when the Raman signal is not detectable. A minimum value of 3.0 is required for the signal to noise. A higher value of the filter $I_{0} / I_{\pi / 2}$ is helpful to increase the credibility $\delta$ in SMSERS verification but decrease the efficiency $\eta$ while a lower value increases the efficiency but lead to a sacrifice in credibility. The SMSERS events are divided into three volumes according to their FWHM of the R6G $1650 \mathrm{~cm}^{-1}$ mode. According to Figure S7b, the FWHM of conventional SERS events are mostly located at $5.8 \mathrm{~cm}^{-1}-7.4 \mathrm{~cm}^{-1}$, the FWHM of SMSERS are mostly located at $3.8 \mathrm{~cm}^{-1}-6.6 \mathrm{~cm}^{-1}$, and the overlapping region is $5.8 \mathrm{~cm}^{-1}-6.6 \mathrm{~cm}^{-1}$. The data comes from the SMSERS events in the manuscript at various concentrations. The results in Figure S8 reveal that the FWHM is narrowing with the increase of the filter, indicating an increase in SMSERS probability. Considering that the FWHM does not change much under the circumstance of $I_{0} / I_{\pi / 2} \geq 5$, the value of the filter is set to be 5.0 in the manuscript after a balance.

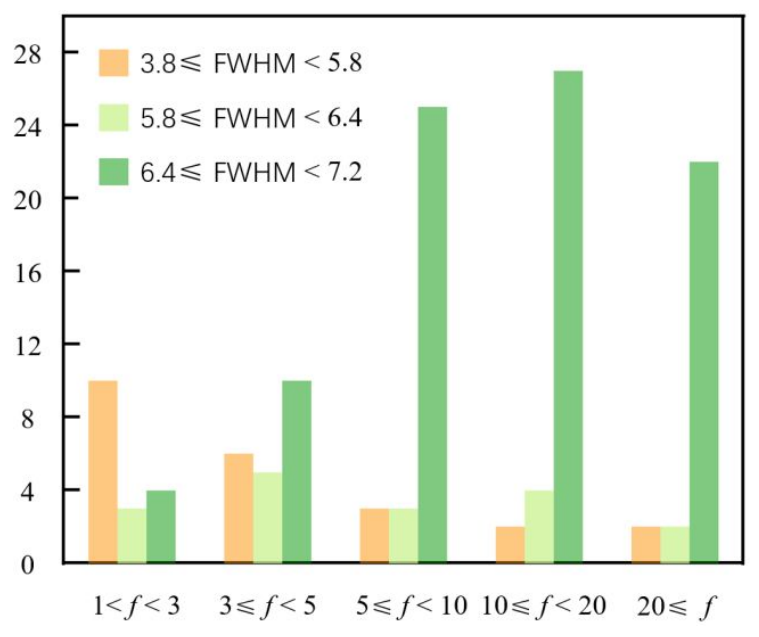

Figure S8. FHWMs distributions with regard to the intensity ratio $f=I_{0} / I_{\pi / 2}$.

References:

1. Hildebrandt, P. and M. Stockburger, Surface-enhanced resonance Raman spectroscopy of Rhodamine $6 G$ adsorbed on colloidal silver. The Journal of Physical Chemistry, 1984. 88(24): p. 5935-5944. 
